\title{
LiFi: transforming fibre into wireless
}

\section{Liang Yin, Mohamed Sufyan Islim, Harald Haas}

Liang Yin, Mohamed Sufyan Islim, Harald Haas, "LiFi: transforming fibre into wireless," Proc. SPIE 10128, Broadband Access Communication Technologies XI, 1012802 (28 January 2017); doi: 10.1117/12.2262047 


\title{
LiFi: Transforming Fibre into Wireless
}

\author{
Liang Yin, Mohamed Sufyan Islim, and Harald Haas \\ Li-Fi Research and Development Centre, Institute for Digital Communications, \\ The University of Edinburgh, King's Buildings, Mayfield Road, Edinburgh, EH9 3JL, U.K. \\ Email: l.yin@ed.ac.uk, m.islim@ed.ac.uk and h.haas@ed.ac.uk
}

\begin{abstract}
Light-fidelity (LiFi) uses energy-efficient light-emitting diodes (LEDs) for high-speed wireless communication, and it has a great potential to be integrated with fibre communication for future gigabit networks. However, by making fibre communication wireless, multiuser interference arises. Traditional methods use orthogonal multiple access (OMA) for interference avoidance. In this paper, multiuser interference is exploited with the use of non-orthogonal multiple access (NOMA) relying on successive interference cancellation (SIC). The residual interference due to imperfect SIC in practical scenarios is characterized with a proportional model. Results show that NOMA offers 5-10 dB gain on the equivalent signal-to-interference-plus-noise ratio (SINR) over OMA. The bit error rate (BER) performance of direct current optical orthogonal frequency division multiplexing (DCO-OFDM) is shown to be significantly improved when SIC is used.
\end{abstract}

Keywords: Light-fidelity (LiFi), visible light communication (VLC), light-emitting diode (LED), non-orthogonal multiple access (NOMA), orthogonal frequency division multiplexing (OFDM).

\section{INTRODUCTION}

Over the past decade, the exponentially growing demand for mobile wireless traffic has motived a number of new technologies for the next generation 5G wireless architecture in order to provide wireless services with higher data rates, lower latency and significantly improved quality of service (QoS). Among them, light-fidelity $(\mathrm{LiFi})^{1-5}$ exploits the unlicensed visible light spectrum (in the vicinity of $430-770 \mathrm{THz}$ ) for bidirectional, high-speed and fully networked wireless communication. Compared to antenna-based radio frequency (RF) technologies, LiFi typically uses existing light-emitting diodes (LEDs) as the signal transmitter, which therefore is more cost-effective and energy-efficient. Experimental works have demonstrated $5 \mathrm{~Gb} / \mathrm{s}$ data rate by using a single Gallium Nitride $\mu$ LED with a maximum optical power of around $3 \mathrm{~mW}{ }^{6}$ Due to existing design and fabrication process, the $3 \mathrm{~dB}$ bandwidth of LEDs typically ranges from 10 to $60 \mathrm{MHz}$. For even higher data rates, laser diodes (LDs) can be used as a promising alternative, and from our recent experimental results it is anticipated that over $100 \mathrm{~Gb} / \mathrm{s}$ data rate can be achieved under standard indoor illumination levels. ${ }^{7}$

Visible light communication (VLC), as a point-to-point communication technique, uses intensity modulation and direct detection (IM/DD) ${ }^{8}$ to transmit data. LiFi differs from VLC in that it stands for a complete wireless networking system including bidirectional communication, multiuser access, user mobility support, handover, etc. Based on the fact that there is a widespread deployment of LED lighting in homes, offices and streetlights, LiFi can be added to existing heterogeneous networks as an additional network layer. The benefit of this is that LiFi can greatly improves the overall network capacity since it receives zero interference from, and adds zero interference to its RF counterparts. Also, since light does not pass through opaque objects, LiFi inherently offers higher information security than RF systems. Earlier works have studied an indoor VLC network integrated with power line communication (PLC). Limited by the achievable data rate of the power lines, only $1 \mathrm{Mb} / \mathrm{s}$ data rate was reported. ${ }^{9}$ Given that there are a large number of LEDs installed in an indoor environment, the capacity of a typical $\mathrm{LiFi}$ network is expected to exceed hundreds of $\mathrm{Gb} / \mathrm{s}$. In this case, optical fibres become a suitable option to be installed as the backbone of high-speed LiFi networks. Fig. 1 gives the schematic diagram of a simplified LiFi network integrated with fibre communication. The infrastructure consists of a central office (CO), which is connected to a metro network and a number of LiFi access points (APs) via optical fibre links. Power cables are connected between a power supply and LiFi APs to provide electricity but not for information transmission.

Broadband Access Communication Technologies XI, edited by Benjamin B. Dingel,

Katsutoshi Tsukamoto, Spiros Mikroulis, Proc. of SPIE Vol. 10128, 1012802

(C) 2017 SPIE · CCC code: $0277-786 X / 17 / \$ 18 \cdot$ doi: 10.1117/12.2262047 


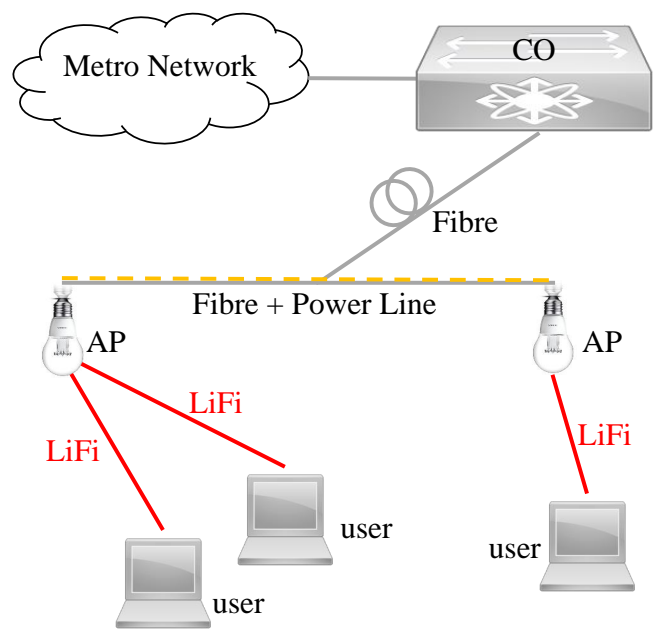

Fig. 1: Schematic diagram of a LiFi network integrated with fibre communication.

Between APs and mobile users are the LiFi link, through which both downlink and uplink communication are conducted, wirelessly. Therefore, LiFi transforms high-speed fibre links into wireless ones.

Compared to metal wires, optical fibres offer a higher signal bandwidth and are able to transmit signals with lower losses. More importantly, optical fibres transmit only light signals, not electricity. Therefore, they are immune to electromagnetic interference, which is a problem that metal wires suffer excessively. Now since LiFi links are used for data communication between LEDs and mobile users, inter-user interference becomes an imminent problem that needs to be addressed in order to fully exploit the capacity of LiFi networks. Traditional methods are based on interference avoidance, such as time/frequency division multiple access (T/FDMA), in which multiuser interference is alleviated by dividing the totally available communication resources among users. However, these methods lead to inefficient use of the already-scarce wireless resources. A user-centric cluster formation technique employing vectored transmission (VT) was proposed in $^{10}$ for efficient interference mitigation in LiFi networks. In this paper, we focus on exploiting the multiuser interference to improve the overall achievable rate of LiFi networks. Our solutions are based on non-orthogonal multiple access (NOMA) with successive interference cancellation (SIC) approach. ${ }^{11,12}$

The rest of the paper is organized as follows. In Sec. 2, a multiuser downlink system model is introduced. SIC with imperfect interference cancellation is studied in Sec. 3, in which the equivalent signal-to-interferenceplus-noise ratio (SINR) is compared between NOMA and OMA. Simulation results are provided in Sec. 4 and conclusions are drawn in Sec. 5.

\section{SYSTEM MODEL}

\subsection{DCO-OFDM}

Multiple variants of optical orthogonal frequency division multiplexing (OFDM) have been proposed for $\mathrm{LiFi}^{13}$ Direct current optical OFDM (DCO-OFDM) is assumed here as the modulation technique, in which signals are transmitted in parallel on a number of orthogonal subcarriers with different central frequencies. Without loss of generality, it is assumed that there are $K$ bit streams to be transmitted to $K$ users. A simplified block diagram illustrating the principle of NOMA is shown in Fig. 2. First, input bit streams for each user are framed and mapped to complex symbols, $X_{k}(l)$, where $k=1, \cdots, K$, based on the selected modulation schemes, e.g. quadrature amplitude modulation (QAM). Here $l$ is the index of the subcarrier. For each OFDM frame, the number of subcarriers used to carry information is equal to $N_{\mathrm{FFT}} / 2-1$, where $N_{\mathrm{FFT}}$ is the size of inverse fast Fourier transform (IFFT) and FFT. This spectral efficiency loss is caused by the Hermitian symmetry constraint $\left(X_{k}(0)=X_{k}\left(N_{\mathrm{FFT}} / 2\right)=0, X_{k}(l)=X_{k}^{*}\left(N_{\mathrm{FFT}}-l\right)\right)$ in order to make the time-domain signal real. This step is 


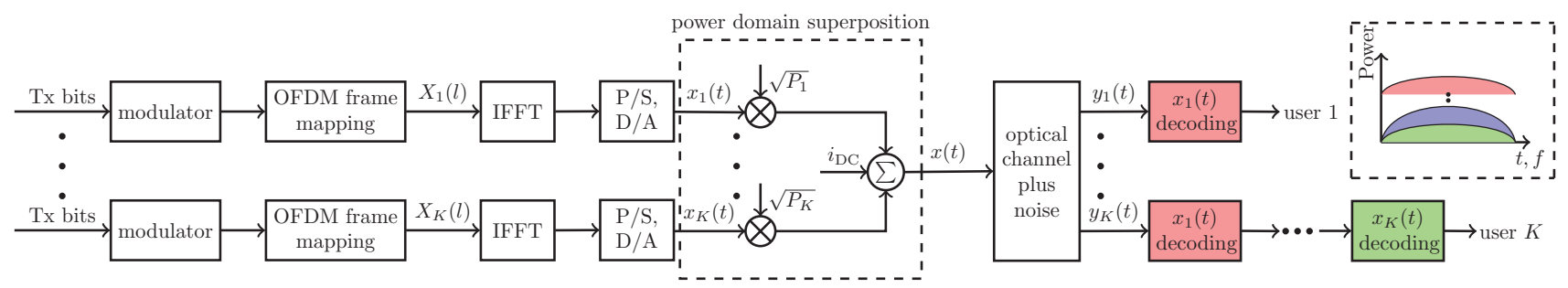

Fig. 2: A simplified block diagram of NOMA-based LiFi network.

necessary since in IM/DD information signals are modulated onto the light intensity, which has to be real and positive, not complex. The time-domain signal $x_{k}(t)$ is obtained by performing the IFFT operation on $X_{k}(l)$ :

$$
x_{k}(t)=\frac{1}{\sqrt{N_{\mathrm{FFT}}}} \sum_{l=0}^{N_{\mathrm{FFT}}-1} X_{k}(l) \exp \left(\frac{j 2 \pi l t}{N}\right) .
$$

For a large number of subcarriers, $x_{k}(t)$ tends to follow a Gaussian distribution, with zero mean and variance $\sigma_{x_{k}}^{2}$. Unlike orthogonal frequency division multiple access (OFDMA) where subcarriers are exclusively assigned to different users, NOMA uses power domain multiplexing to realize MA so that all of the users can use the whole subcarriers. Specifically, each user is allocated with signal power $P_{k}$, and the superimposed signal to be transmitted by the LiFi AP is:

$$
\begin{aligned}
x(t) & =\sum_{k=1}^{K} \sqrt{P_{k}} x_{k}(t)+i_{\mathrm{DC}} \\
& =\frac{1}{\sqrt{N_{\mathrm{FFT}}}} \sum_{k=1}^{K} \sum_{l=0}^{N_{\mathrm{FFT}}-1} \sqrt{P_{k}} X_{k}(l) \exp \left(\frac{j 2 \pi l t}{N_{\mathrm{FFT}}}\right)+i_{\mathrm{DC}},
\end{aligned}
$$

where $i_{\mathrm{DC}}$ is the DC bias added to the LED in order to ensure a positive instantaneous intensity. The output of Eq. (2) is given by:

$$
\tilde{\mathbf{x}}=\left[x(0), x(1), \ldots, x\left(N_{\mathrm{FFT}}-1\right)\right]^{T} .
$$

A cyclic prefix $(\mathrm{CP})$ of length $N_{\mathrm{CP}}-1$ is then inserted at the start of each OFDM frame. This CP is composed of the last $N_{\mathrm{CP}}-1$ symbols of each frame:

$$
\mathbf{x}=\left[x\left(N_{\mathrm{FFT}}-N_{\mathrm{CP}}+1\right), \ldots, x\left(N_{\mathrm{FFT}}-2\right), x\left(N_{\mathrm{FFT}}-1\right), x(0), x(1), \ldots, x\left(N_{\mathrm{FFT}}-1\right)\right]^{T} .
$$

The cyclic prefix is required to be larger than the maximum delay spread of the channel $T_{\mathrm{d}}$ in order for the receiver to avoid inter-symbol interference (ISI), since the channel dispersion affects only the first $N_{\mathrm{CP}}-1$ symbols. With the insertion of CP, the spectral efficiency of DCO-OFDM is given by:

$$
\eta_{\mathrm{DCO}}=\frac{\log _{2}(M)\left(N_{\mathrm{FFT}}-2\right)}{2\left(N_{\mathrm{FFT}}-N_{\mathrm{CP}}\right)}
$$

where $M$ is the constellation size of the QAM symbol used.

It is easy to show that $x(t)$ in Eq. (2) also follows the Gaussian distribution with mean value $i_{\mathrm{DC}}$ and variance $\sum_{k=1}^{K} P_{k} \sigma_{x_{k}}^{2}$. Due to the total power constraint, $\sum_{k=1}^{K} P_{k}=P_{\mathrm{tx}}$ should be satisfied, where $P_{\mathrm{tx}}$ denotes the total available electrical power for signal transmission. To ensure successful interference cancellation in NOMA, users with lower channel gains are generally allocated with more signal power. Therefore, by denoting the channel gains for $K$ users as $h_{1} \leq \cdots \leq h_{K}$, we have $P_{1} \geq \cdots \geq P_{K}$. 


\subsection{LiFi Channel}

For downlink transmission, where the LiFi AP of interest is placed at the origin and $K$ users are uniformly distributed underneath within a circular area of radius $r_{\mathrm{e}}$. The vertical distance between the LiFi AP and users is denoted by $L$. The AP is assumed to be facing vertically downward and the users are assumed to be facing vertically upward with a field-of-view (FOV) of $\Psi_{\text {fov }}$. The LiFi channel can be characterized by its dominant line-of-sight (LOS) path, whose propagation gain is given by: ${ }^{14}$

$$
h_{k}=\frac{(m+1) A R_{\mathrm{p}}}{2 \pi d_{k}^{2}} \cos ^{m}\left(\phi_{k}\right) T\left(\psi_{k}\right) g\left(\psi_{k}\right) \cos \left(\psi_{k}\right),
$$

where $m=-1 / \log _{2}\left(\cos \left(\Phi_{1 / 2}\right)\right)$ is the Lambertian order of the LED and $\Phi_{1 / 2}$ denotes its semi-angle; $A$ denotes the detection area of the PD and $R_{\mathrm{p}}$ denotes its responsivity; $d_{k}$ is the Euclidean distance between the LED and the user; $\phi_{k}$ is the angle of irradiance; $\psi_{k}$ is the angle of incidence; $T\left(\psi_{k}\right)$ represents the gain of the optical filter used at the receiver; and $g\left(\psi_{k}\right)$ represents the gain of the optical concentrator, given by: ${ }^{14}$

$$
g\left(\psi_{k}\right)=\left\{\begin{array}{cc}
\frac{n^{2}}{\sin ^{2}\left(\Psi_{\text {fov }}\right)}, & 0 \leq \psi_{k} \leq \Psi_{\text {fov }} \\
0, & \psi_{k}>\Psi_{\text {fov }}
\end{array}\right.
$$

where $n$ is the reflective index of the optical concentrator used at the receiver front-end. After propagating through free space, the received signal at the $k$-th user can be expressed as:

$$
y_{k}(t)=h_{k} x(t)+n_{k}(t)
$$

where $n_{k}(t)$ is the real-valued Gaussian noise with zero mean and single-sided variance $N_{0}$.

\section{SUCCESSIVE INTERFERENCE CANCELLATION}

Before detecting its own signal, SIC is performed at each user to remove the signals that are intended for other users with lower channel gains. Since the signal intended for user 1 has the highest power, the SIC process begins by detecting and canceling signal $x_{1}(t)$ at each user. As a result, the SINR of user $k$ during its first SIC process is given by:

$$
\gamma_{k}(1)=\frac{P_{1}}{\sum_{u=2}^{K} P_{u}+N_{0} / h_{k}^{2}}
$$

If $\gamma_{k}(1)$ is greater than a threshold $b$, it is assumed that the receiver is able to detect and subtract this signal from the received aggregate signal before the next SIC process. However, in the SIC process, it requires the receiver to reconstruct the waveform of the decoded users. Since this operation involves channel estimation as well as signal decoding, that can never be perfect in practice, there exist interference cancellation errors. Therefore, residual interference exists in the remaining part of the signal, and this unavoidably degrades the performance of the next SIC process. Such imperfect interference cancellation process can be characterized with a proportional model, ${ }^{15}$ in which it is assumed that the cancellation of a signal with power $P$ can only cancel a portion of the signal and leaves a residual interference power of $z P$, with $z \leq 1$. Given that the first SIC process at user $k$ is successful, the SINR for the second SIC process is:

$$
\gamma_{k}(2)=\frac{P_{2}}{z P_{1}+\sum_{u=3}^{K} P_{u}+N_{0} / h_{k}^{2}} .
$$

Again, the second SIC process is successful if $\gamma_{k}(2)>b$, and unsuccessful otherwise. Given that the $(s-1)$-th SIC process is successful, the SINR of the $s$-th SIC is:

$$
\gamma_{k}(s)=\frac{P_{s}}{z \sum_{u=1}^{s-1} P_{u}+\sum_{v=s+1}^{K} P_{v}+N_{0} / h_{k}^{2}}
$$




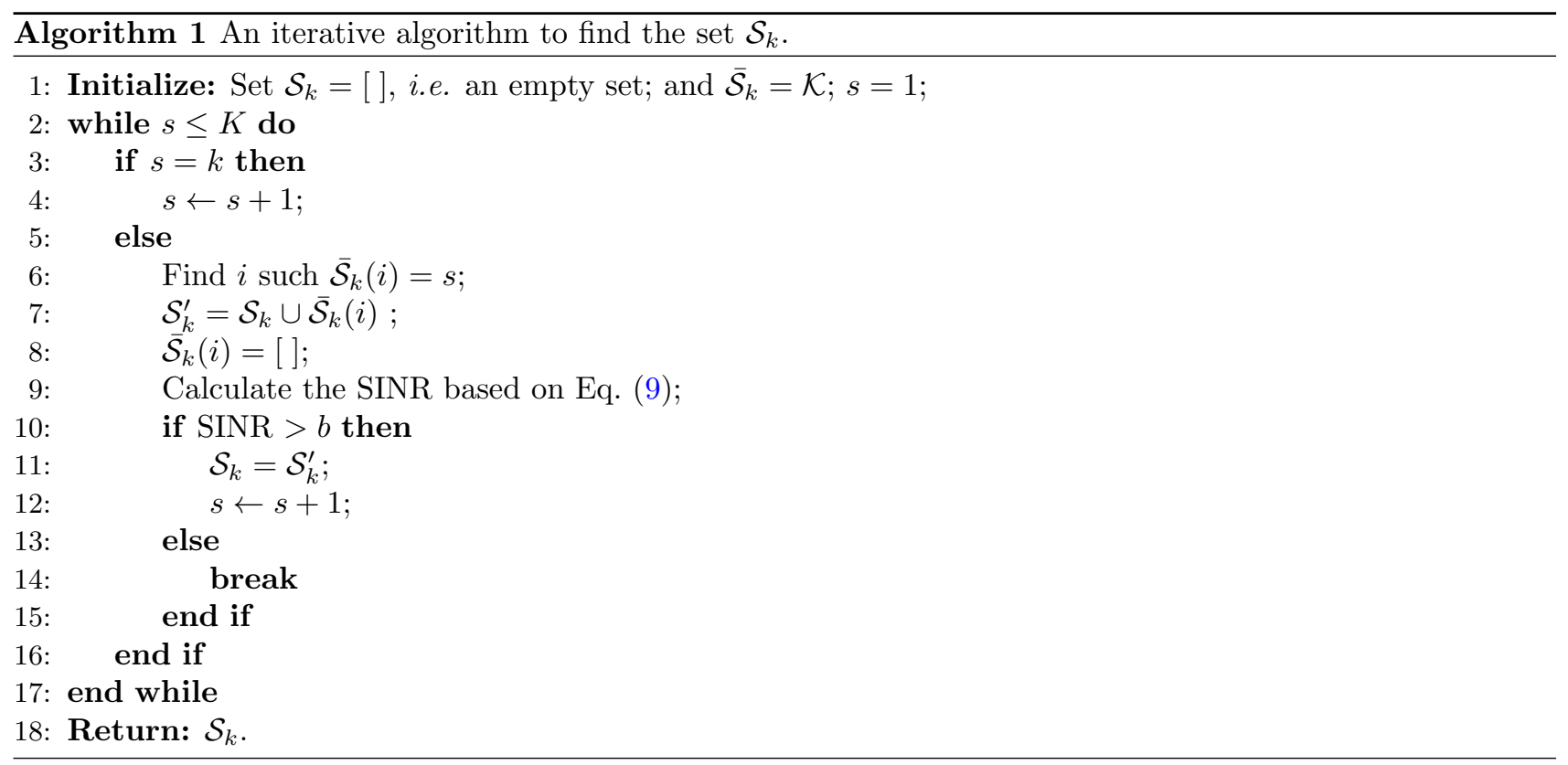

where $s=2, \cdots, k-1, k+1, \cdots, K$. The SIC process repeats by increasing the value of $s$ until $\gamma_{k}(s) \leq b$. At the end of SIC process, the SINR for user $k$ is finally given by:

$$
\gamma_{k}=\left\{\begin{array}{cc}
\frac{P_{k}}{\sum_{u \in \mathcal{K} \backslash k} P_{u}+N_{0} / h_{k}^{2}}, & s=1 \\
\frac{\sum_{u \in \mathcal{S}_{k}} P_{u}+\sum_{v \in \mathcal{K} \backslash \mathcal{S}_{k} \backslash k} P_{k}}{P_{v}+N_{0} / h_{k}^{2}}, & s>1,
\end{array},\right.
$$

where $\mathcal{K}$ denotes the set which includes all of the users and $\mathcal{S}_{k}$ denotes the set including users whose signals have be detected and canceled by user $k$ during the SIC process, i.e. $\gamma_{k}\left(\mathcal{S}_{k}\right)>$ b. An iterative algorithm to find $\mathcal{S}_{k}$ is described in Algorithm 1, in which $\overline{\mathcal{S}}_{k}$ denotes the complement of set $\mathcal{S}_{k}$.

In OMA, signals for different users are transmitted using orthogonal resources so that the interference term does not exist. The SINR, which is equivalent to signal-to-noise ratio (SNR) in this case, is given by:

$$
\gamma_{k}=\frac{P_{k}}{N_{0} / h_{k}^{2}} .
$$

To allow a fair comparison between OMA and NOMA, we define an equivalent SINR for the OMA case. The equivalent SINR, denoted by $\tilde{\gamma}_{k}$, is related to the SINR by the following equation:

$$
\frac{1}{K} \log _{2}\left(1+\gamma_{k}\right)=\log _{2}\left(1+\tilde{\gamma}_{k}\right) .
$$

The equivalent SINR can be solved as:

$$
\tilde{\gamma}_{k}=\left(1+\gamma_{k}\right)^{1 / K}-1 .
$$

Note that in OMA the equivalent SINR is equal to the actual SINR in the single user scenario, i.e. $K=1$. As the number of users increases, the equivalent SINR drops exponentially, which therefore limits the total capacity of the network. These results are also shown in Fig. 3. For example, assuming a LiFi network with 8 users, the equivalent SINR is around $0 \mathrm{~dB}$ when the actual SINR is $25 \mathrm{~dB}$. 


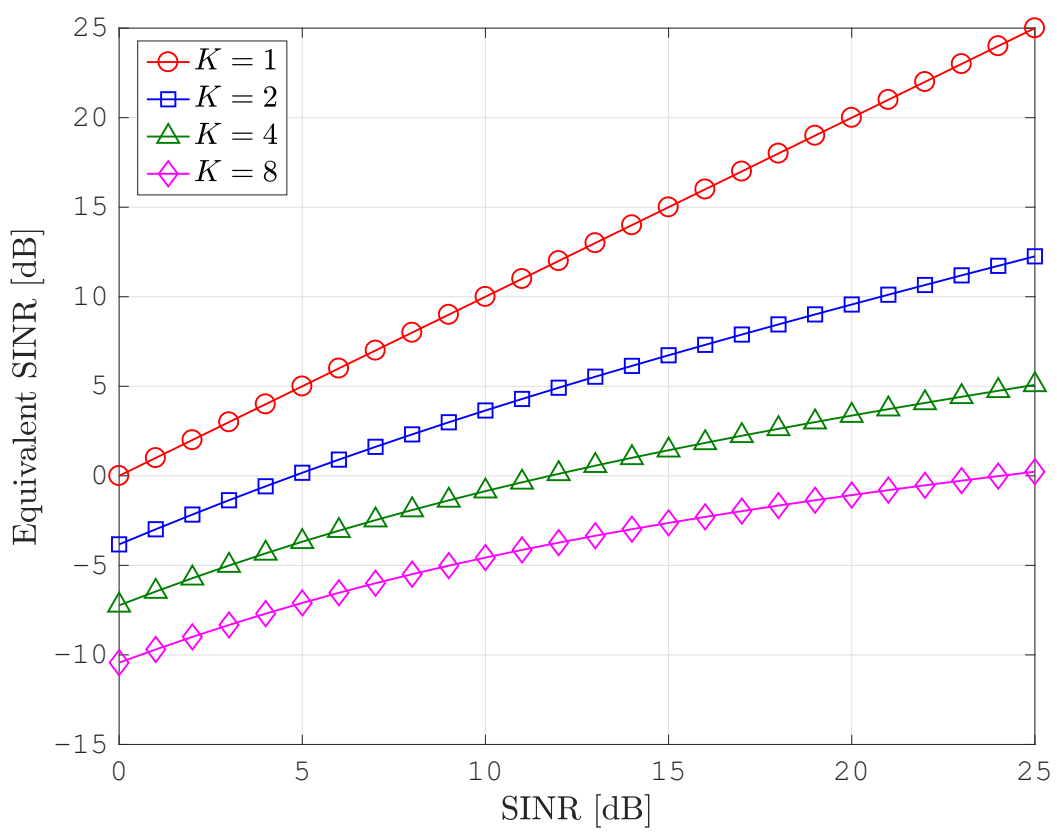

Fig. 3: Equivalent SINR as a function of SINR.

Table 1: Simulation parameters

\begin{tabular}{l|r}
\hline Parameter name, notation & value \\
\hline Cell Height, $L$ & $2.15 \mathrm{~m}$ \\
Cell radius, $r_{\mathrm{e}}$ & $3.6 \mathrm{~m}$ \\
LED semi-angle, $\Phi_{1 / 2}$ & $60^{\circ}$ \\
Total signal power, $P_{\text {elec }}$ & $1 \mathrm{~W}$ \\
Receiver FoV, $\Psi_{\text {fov }}$ & $60^{\circ}$ \\
Receiver responsivity, $R_{\mathrm{p}}$ & $0.4 \mathrm{~A} / \mathrm{W}$ \\
Receiver detection area, $A$ & $1 \mathrm{~cm}^{2}$ \\
Reflective index, $n$ & 1.5 \\
Optical filter gain, $T$ & 1 \\
Signal bandwidth, $B$ & $20 \mathrm{MHz}$ \\
Noise power spectral density & $\mathrm{A}^{2} / \mathrm{Hz}$ \\
Relative residual interference power after SIC, $z$ & 0.01 \\
SINR threshold for successful SIC, $b$ & 0.1 \\
OFDM frame size, $N_{\text {FFT }}$ & 512 \\
$M$-QAM constellation size, $M$ & 4 \\
\hline
\end{tabular}

\section{SIMULATION RESULTS}

In this section, Monte Carlo simulations are conducted to evaluate the performance of NOMA in a multiuser LiFi network. The performance metrics under investigation are the cumulative distribution function (CDF) of the equivalent SINR and the achieved bit error rate (BER) using DCO-OFDM at each user. The parameters used for the simulation setup are summarized in Table. 1, if not otherwise specified.

\subsection{CDF of the Equivalent SINR}

By assuming uniformly distributed mobile users, the empirical CDF of the equivalent SINR for both NOMA and OMA are obtained from a total number of 10,000 realizations, as shown in Fig. 4. An exponential power allocation scheme is assumed, which gives the following power coefficients: $\left[P_{1}, P_{2}, P_{3}\right]=[0.8,0.15,0.05]$. Note that other power allocation values are possible, and the optimal power allocation will be considered in future 


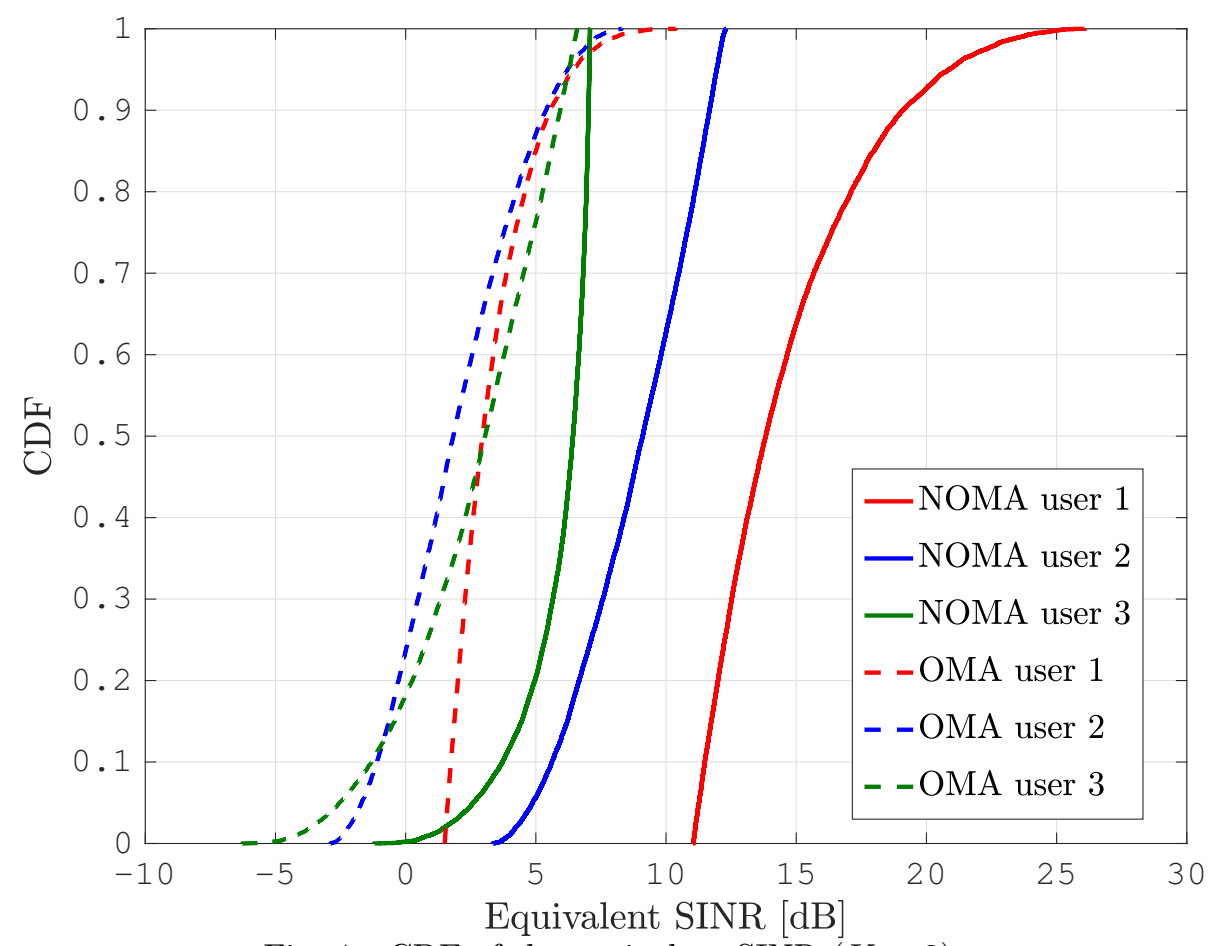

Fig. 4: $\mathrm{CDF}$ of the equivalent $\operatorname{SINR}(K=3)$.

research. Note that although there is no actual interference in the OMA case, the total available communication resources have to be divided among all of the users, which in principle degrades the throughput of the network. Therefore, the equivalent SINR, instead of SNR, is computed in order to provide a fair comparison between OMA and NOMA. It can be seen that, with interference cancellation NOMA can achieve $5-10 \mathrm{~dB}$ improvement on the equivalent SINR over OMA. A higher SINR gain is expected if the number of users in the LiFi network increases.

\subsection{BER Performance}

The BER versus SINR performance of the proposed system is also investigated using Monte Carlo simulations. To allow a fair comparison among users, the results are reported for the electrical SINR after ignoring the DC bias. The effect of different power allocations on the BER performance of a two-user LiFi network is presented in Fig. 5. It can be shown, that the system performance for both users improves as the power ratio $P_{1} / P_{2}$ increases. The power at user 2 is given by $P_{2}=P_{\mathrm{tx}}-P_{1}$. The worst case scenario for SIC is shown at equal power allocation. The BER performance of user 2 is shown to outperform the BER performance of user 1, which is expected as the proposed SIC algorithm insures that most of the interference caused by user 1 is cancelled. However, user 1 has a higher SINR, which means that the BER performance of user 1 will be affected by all of the interference caused by user 2 .

The BER performance of a three-user LiFi network is presented in Fig. 6. The power allocation coefficients are obtained from the same exponential power allocation model reported above. It can be shown that the performance of user 1 is unaffected by the proposed algorithm as user 1 has the highest SINR. The performances of other users are significantly improved compared to the case when SIC is not applied. The BER floor of $10^{-4}$ is considered because it can allow for forward error correction codes to be used. ${ }^{16}$ This BER can be achieved for the proposed algorithm at SINR values of $17.53 \mathrm{~dB}, 15.41 \mathrm{~dB}$, and $10.71 \mathrm{~dB}$ for user 1, user 2, and user 3, respectively. When SIC is not used, the BER floor for both user 2 and user 3 is around 0.5 for all the considered SINR values. 


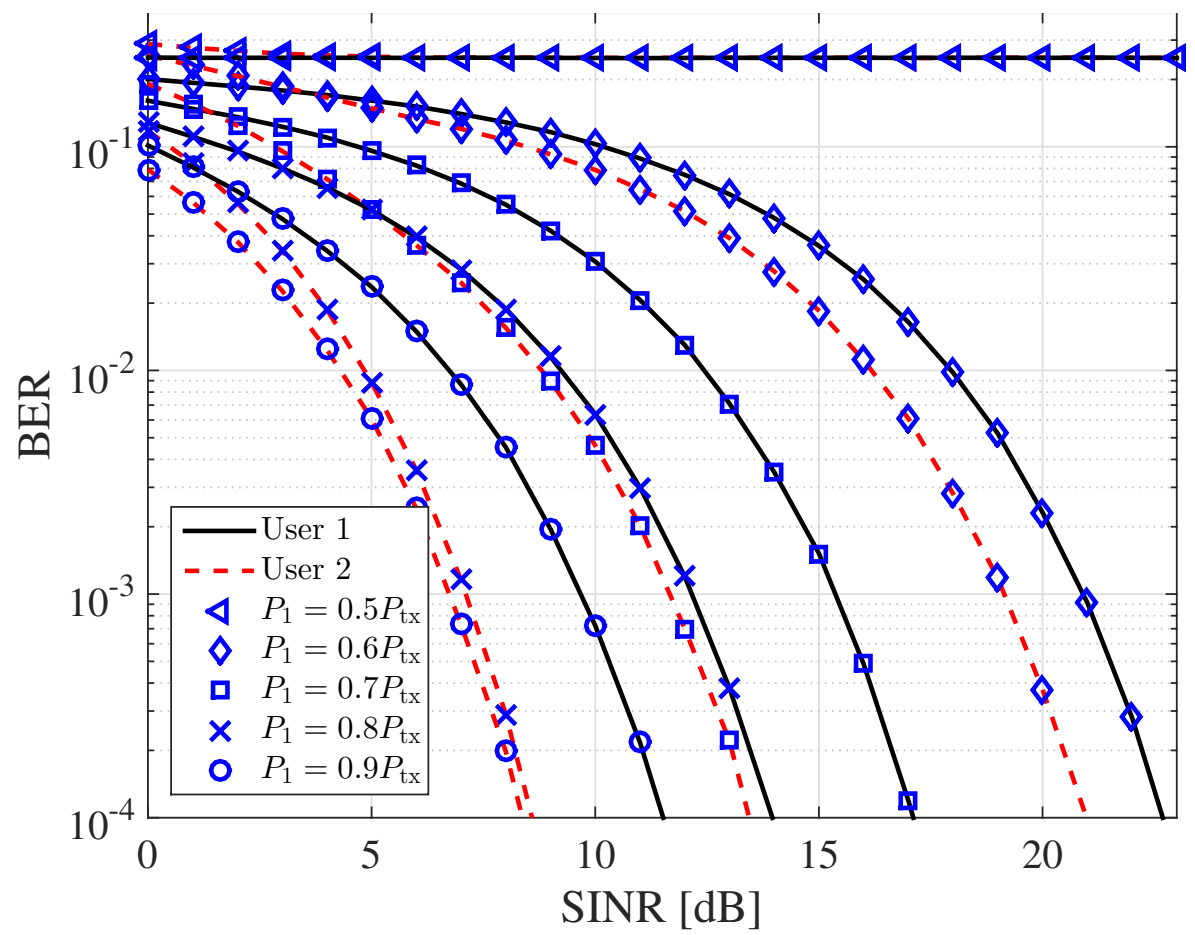

Fig. 5: BER versus SINR performance for a two-user network using different power allocation sets.

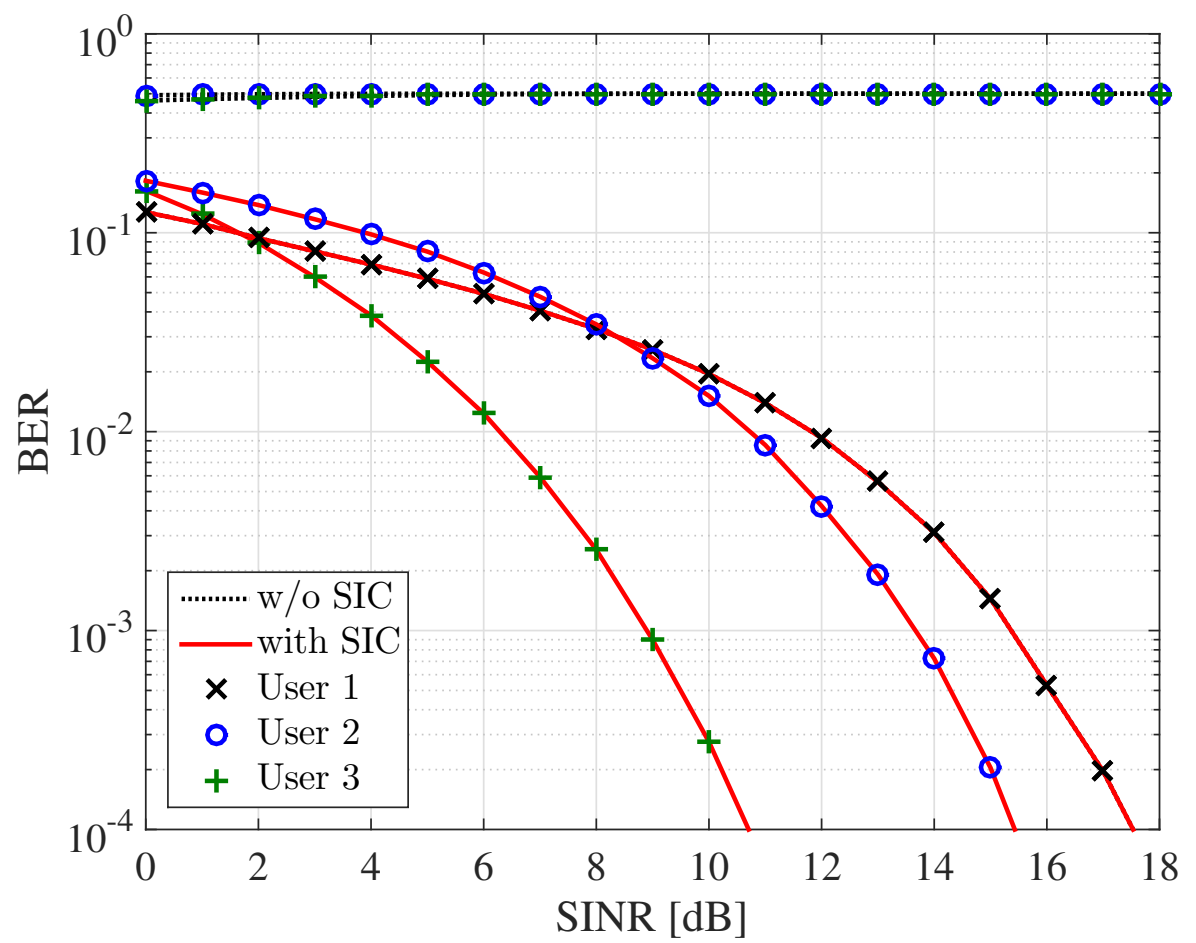

Fig. 6: BER versus SINR system performance for a three-user network.

\section{CONCLUSION}

In this paper we investigated the multiuser access problem in LiFi networks. Our solutions are based on NOMA with power domain multiplexing to transmit multi-stream data to multiple users using the same time/frequency 
resources. A proportional model is used to characterize the residual interference due to imperfect interference cancellation in practical scenarios. Results show that, compared with OMA, NOMA provides $5-10 \mathrm{~dB}$ improvement on the equivalent SINR and hence increases the overall throughput of LiFi networks. The BER performance is improved significantly compared to the case when the proposed algorithm is not used. These results indicate that LiFi technology is a promising candidate for future gigabit wireless networks.

\section{ACKNOWLEDGMENTS}

Professor Harald Haas acknowledges support by the UK Engineering and Physical Sciences Research Council (EPSRC) under Grant EP/K008757/1.

\section{REFERENCES}

[1] Haas, H., "High-Speed Wireless Networking Using Visible Light," SPIE Newsroom (Apr. 2013).

[2] Haas, H., Yin, L., Wang, Y., and Chen, C., "What is LiFi?," J. Lightw. Technol. 34, 1533-1544 (Mar. 2016).

[3] Wu, S., Wang, H., and Youn, C. H., "Visible Light Communications for 5G Wireless Networking Systems: From Fixed to Mobile Communications ," IEEE Netw. 28, 41-45 (Nov. 2014).

[4] Grubor, J., Randel, S., Langer, K. D., and Walewski, J., "Broadband Information Broadcasting Using LED-Based Interior Lighting," J. Lightw. Technol. 26, 3883-3892 (Dec. 2008).

[5] O’Brien, D., Zeng, L., Le-Minh, H., Faulkner, G., Walewski, J. W., and Randel, S., "Visible Light Communications: Challenges and Possibilities," in [Proc. IEEE 19th Int. Symp. Pers. Indoor Mobile Radio Commun. (PIMRC)], 1-5 (Sept. 2008).

[6] Ferreira, R. X. G., Xie, E., McKendry, J. J. D., Rajbhandari, S., Chun, H., Faulkner, G., Watson, S., Kelly, A. E., Gu, E., Penty, R. V., White, I. H., OBrien, D. C., and Dawson, M. D., "High bandwidth gan-based micro-leds for multi-gb/s visible light communications," IEEE Photonics Technology Letters 28, 2023-2026 (Oct 2016).

[7] Tsonev, D., Videv, S., and Haas, H., "Towards a 100 Gb/s Visible Light Wireless Access Network," Opt. Express 23, 1627-1637 (Jan 2015).

[8] Zhang, D. and Hranilovic, S., "Bandlimited Optical Intensity Modulation Under Average and Peak Power Constraints," IEEE Trans. Commun. 64, 3820-3830 (Sept. 2016).

[9] Komine, T. and Nakagawa, M., "Integrated System of White LED Visible-Light Communication and PowerLine Communication," IEEE Trans. Consum. Electron. 49(1), 71-79 (2003).

[10] Li, X., Jin, F., Zhang, R., Wang, J., Xu, Z., and Hanzo, L., "Users First: User-Centric Cluster Formation for Interference-Mitigation in Visible-Light Networks," IEEE Trans. Wireless Commun. 15, 39-53 (Jan. 2016).

[11] Yin, L., Wu, X., and Haas, H., "On the Performance of Non-Orthogonal Multiple Access in Visible Light Communication," in [Proc. IEEE 26th Annual Symposium on Personal, Indoor and Mobile Radio Communications (PIMRC)], 1376-1381 (Sept. 2015).

[12] Yin, L., Popoola, W. O., Wu, X., and Haas, H., "Performance Evaluation of Non-Orthogonal Multiple Access in Visible Light Communication," IEEE Trans. Commun. pp, 1-1 (Sept. 2016).

[13] Islim, M. S. and Haas, H., "Modulation Techniques for Li-Fi," ZTE Communications 14, 29-40 (April 2016).

[14] Kahn, J. and Barry, J., "Wireless Infrared Communications," Proc. IEEE 85, 265-298 (Feb. 1997).

[15] Weber, S. P., Andrews, J. G., Yang, X., and de Veciana, G., "Transmission Capacity of Wireless ad hoc Networks With Successive Interference Cancellation," IEEE Trans. Inf. Theory 53, 2799-2814 (Aug. 2007).

[16] ITU-T, "Forward Error Correction for High Bit-Rate DWDM Submarine Systems," Tech. Rep. ITU-T G.975.1, ITU (Retrieved Nov. 19, 2013 from http://www.itu.int/rec/T-REC-G.975.1-200402-I/en, 2004). 